\title{
Categorias de Necessidades Educacionais Especiais Enquanto Preditoras de Déficits em Habilidades Sociais na Infância
}

\author{
Categories of Special Educational Needs as Predictors of Social Skills Deficits \\ in Childhood
}

\author{
Lucas Cordeiro Freitas ${ }^{*, a} \&$ Zilda Aparecida Pereira Del Prette \\ ${ }^{a}$ Universidade Federal de Alagoas, Maceió, AL, Brasil \\ $\&{ }^{b}$ Universidade Federal de São Carlos, São Carlos, SP, Brasil
}

\begin{abstract}
Resumo
Esta pesquisa verificou a força preditiva de 12 categorias de necessidades educacionais especiais sobre o repertório de habilidades sociais de crianças: Autismo, Deficiência Auditiva, Deficiência Intelectual Leve, Deficiência Intelectual Moderada, Deficiência Visual, Desvio Fonológico, Dificuldades de Aprendizagem, Dotação e Talento, Problemas de Comportamento Externalizantes, Problemas de Comportamento Internalizantes, Problemas de Comportamento Internalizantes e Externalizantes e TDAH. Cento e vinte estudantes de escolas regulares e especiais, com idades entre seis e 15 anos, foram avaliados por seus professores por meio do Sistema de Avaliação de Habilidades Sociais (SSRS-BR). As necessidades especiais mais fortemente preditoras para déficits de habilidades sociais foram: TDAH, Problemas de Comportamento Misto, Autismo, Problemas Externalizantes, Problemas Internalizantes e Dificuldades de Aprendizagem. Discutem-se as características específicas dessas categorias e as necessidades de aprimoramento de seu repertório de habilidades sociais.

Palavras-chave: Habilidades sociais, necessidades educacionais especiais, avaliação psicológica, regressão estatística.
\end{abstract}

\begin{abstract}
This study verified the predictive force of 12 categories of special educational needs on the social skills repertoire of children: Autism, Hearing Impairment, Mild Intellectual Disabilities, Moderate Intellectual Disabilities, Visual Impairment, Phonological Disorder, Learning Disabilities, Giftedness and Talent, Externalizing Behavior Problems, Internalizing Behavior Problems, Internalizing and Externalizing Behavior Problems and Attention Deficit Hyperactivity Disorder. One hundred and twenty students from regular and special schools, aged between six and 15 years, were assessed by their teachers using the Social Skills Rating System. The special needs which strongly predicted social skills deficits were: Attention Deficit Hyperactivity Disorder, Internalizing and Externalizing Behavior Problems, Autism, Externalizing Behavior Problems, Internalizing Behavior Problems and Learning Disabilities. Specific characteristics of these categories and the needs to improve their social skills repertoire are discussed.

Keywords: Social skills, special educational needs, psychological assessment, statistical regression.
\end{abstract}

As habilidades sociais podem ser definidas como o conjunto de comportamentos sociais do repertório de um indivíduo que favorecem o relacionamento saudável e produtivo com as demais pessoas (Del Prette \& Del Prette, 2005a, 2009). Trata-se de um conjunto de comportamentos, que inclui as classes de cooperação, comunicação, assertividade, empatia, autocontrole, que contribuem para a competência social e, portanto, ampliam a probabilida- de de maximizar reforçadores e minimizar estimulação aversiva para o indivíduo (Del Prette \& Del Prette, 2009, 2010/2012).

As habilidades sociais vêm sendo cada vez mais reconhecidas como um fator favorável ao desenvolvimento social e acadêmico infantil, tanto em estudos transversais quanto longitudinais (Caprara, Barbaranelli, Pastorelli, Bandura, \& Zimbardo, 2000; Del Prette, Del Prette, Oliveira, Gresham, \& Vance, 2012; DiPerna \& Elliott, 1999; Gresham \& Elliott, 2008). Além disso, elas se incluem entre as classes de condutas adaptativas (Gresham \& Elliott, 1987), consideradas relevantes para os diferentes quadros de necessidades especiais, configurando-se, inclusive, como um dos critérios diagnósticos de algumas deficiências e transtornos, particularmente na deficiência 
Freitas, L. C. \& Del Prette, Z. A. P. (2014). Categorias de Necessidades Educacionais Especiais Enquanto Preditoras de Déficits em Habilidades Sociais na Infância.

intelectual (Schalock et al., 2010) e no autismo (American Psychiatric Association [APA], 2002). Essa qualificação é apoiada por fortes evidências, na literatura, de associações positivas entre um repertório elaborado de habilidades sociais e diversos indicadores de funcionamento adaptativo, tais como bom desempenho acadêmico (Bandeira, Rocha, Pires, Del Prette, \& Del Prette, 2006), status social positivo no grupo (Molina \& Del Prette, 2007) e menor frequência de problemas de comportamento internalizantes (Rubin, Coplan, \& Bowker, 2009) e externalizantes (Sorlie, Hagen, \& Ogden, 2008).

Os estudos da área do Treinamento de Habilidades Sociais (THS) com crianças têm sido realizados com uma ampla variedade de populações que se caracterizam como usuárias dos recursos da Educação Especial, tais como alunos com deficiências intelectual, auditiva, visual, hiperatividade, autismo, dentre outros (Del Prette \& Del Prette, 2009). Os resultados evidenciam que, em geral, existe um comprometimento de habilidades sociais nas diferentes categorias de crianças e adolescentes com necessidades educacionais especiais (Angélico \& Del Prette, 2011; Barreto, Freitas, \& Del Prette, 2011; Costa \& Del Prette, 2012; Gresham, Lane, MacMillan, \& Bocian, 1999; Thomas, Shapiro, DuPaul, Lutz, \& Kern, 2011; Wauters \& Knoors, 2008). Uma exceção a essa tendência pode ser observada somente em crianças dotadas e talentosas, ainda que os resultados produzidos pelos estudos de caracterização do repertório social dessa população permaneçam contraditórios e inconclusivos (Bain \& Bell, 2004; Lehman \& Erdwins, 2004; Versteynen, 2001).

$\mathrm{O}$ conceito de necessidades educacionais especiais começou a ser utilizado a partir da década de 60 , trazendo mudanças no que se refere à identificação e às intervenções educacionais voltadas para as crianças que apresentam problemas na aprendizagem (Marchesi, 2004). Uma das vantagens apontadas por Marchesi (2004) na utilização do termo necessidades educacionais especiais é o seu caráter relativista e contextual, na medida em que define que os alunos com deficiências ou dificuldades de aprendizagem podem apresentar diferentes necessidades educacionais ao longo de sua escolarização, remetendo à provisão de recursos específicos para atender a tais necessidades. Existem características próprias a cada tipo de específico de limitação apresentada pela criança que podem ajudar a proporcionar a intervenção educacional mais conveniente em cada caso (Marchesi, 2004).

Podem ser encontradas na literatura diversas tentativas de se agrupar as populações com necessidades especiais em categorias mais específicas, como é o caso dos manuais médicos e psiquiátricos como o CID-10 (Organização Mundial da Saúde, 1997) e o DSM-IV-TR (APA, 2002). No entanto, optou-se por utilizar algum sistema de classificação que enfocasse mais os aspectos educacionais das populações com necessidades especiais e, de acordo com esse critério, foram levantadas algumas classificações brasileiras e internacionais reconhecidas dentro do campo da Educação Especial.
Algumas classificações nacionais apresentam definições relativamente genéricas com relação às populações atendidas pela Educação Especial, como é o caso da Política Nacional de Educação Especial na Perspectiva da Educação Inclusiva (Ministério da Educação [MEC], 2007), na qual apenas são consideradas as pessoas com deficiência física, intelectual ou sensorial, com transtornos globais do desenvolvimento (incluindo síndromes do espectro do autismo e psicose infantil) e pessoas com altas habilidades/superdotação. Nessa classificação não são considerados aqueles alunos que apresentam dificuldades de aprendizagem ou problemas de comportamento não associados a deficiências ou quadros clínicos.

Nesse sentido, elegeu-se como norteadora deste trabalho a classificação das necessidades educacionais especiais contida na lei americana Individuals with Disabilities Education Improvement Act (IDEA, 2004). Ainda que construída em um contexto cultural diferente e reconhecendo-se que muitas das questões contidas nessa lei não podem ser transpostas diretamente para a realidade brasileira, entende-se que a classificação proposta ampliou o quadro das populações que podem ser atendidas pela Educação Especial e, ao mesmo tempo, especificou com maiores detalhes as características das populações de forma a facilitar a identificação e as propostas educacionais diferenciadas. A IDEA propõe a classificação das crianças com necessidades educacionais especiais em 13 categorias: (a) Autismo, (b) Surdocegueira, (c) Surdez, (d) Distúrbio Emocional (que inclui problemas de comportamento externalizantes e internalizantes), (e) Deficiência Auditiva, (f) Retardo Mental, (g) Deficiências Múltiplas, (h) Deficiência Física, (i) Outras Condições de Saúde (incluindo Transtorno de Déficit de Atenção e Hiperatividade), (j) Dificuldade de Aprendizagem Específica, (k) Distúrbio de Fala ou Linguagem, (1) Traumatismo Cranioencefálico e (m) Deficiência Visual (incluindo Cegueira).

Com base nos estudos da literatura em Educação Especial e nos aportes teóricos do campo das Habilidades Sociais (Del Prette \& Del Prette, 2005a, 2010/2012; Elliott \& Gresham, 2008; Feitosa, Del Prette, \& Del Prette, 2012), é razoável supor que, teoricamente, as necessidades especiais constituam-se como um fator de risco para déficits em habilidades sociais, e que, empiricamente, configurem-se como preditoras de dificuldades comportamentais na área de desenvolvimento socioemocional. São considerados fatores de risco as características individuais ou ambientais que dificultam o desenvolvimento da criança e favorecem o surgimento de problemas de ordem física, emocional ou social (Price \& Zwolinski, 2010).

Entende-se que a realização de estudos de avaliação do repertório de crianças com diferentes necessidades educacionais especiais, utilizando delineamentos multivariados de investigação (Abbad \& Torres, 2002; Tabachnick \& Fidell, 2007), poderia contribuir para uma melhor compreensão a respeito do impacto específico de cada deficiência sobre as habilidades sociais. Pode-se hipotetizar que diferentes grupos de 
crianças com necessidades especiais estão expostos a diferentes contingências de reforçamento, em função das limitações orgânicas, físicas e sensoriais e também de seu comportamento social atípico ou mais restrito. Entende-se que as características orgânicas específicas de algumas categorias de necessidades especiais, conjugadas aos fatores ambientais, poderiam produzir impactos de diferentes magnitudes sobre o repertório de habilidades sociais das crianças, o que por sua vez, tem consequências sobre o seu desenvolvimento. Entretanto, ainda que plausível, essa hipótese não vem sendo suficientemente explorada nos estudos da literatura revisados, principalmente se se considerar o conjunto das diferentes necessidades educacionais existentes.

O estudo de L. C. Freitas e Del Prette (2013) realizado com a mesma amostra do presente artigo, comparou, com base na avaliação do professor, o repertório de habilidades sociais de crianças pertencentes a 12 grupos de necessidades educacionais especiais (Autismo, Deficiência Auditiva, Deficiência Intelectual Leve, Deficiência Intelectual Moderada, Deficiência Visual, Desvio Fonológico, Dificuldades de Aprendizagem, Dotação e Talento, Problemas de Comportamento Externalizantes, Problemas de Comportamento Internalizantes, Problemas de Comportamento Internalizantes e Externalizantes e Transtorno de Déficit de Atenção e Hiperatividade-TDAH) utilizando-se análises de variância (ANOVA). As crianças com TDAH, Autismo, Problemas de Comportamento Misto, Problemas Externalizantes, Problemas Internalizantes e Dificuldades de Aprendizagem obtiveram escores médios significativamente mais baixos de habilidades sociais na avaliação dos professores.

Em complemento ao estudo anterior, a presente pesquisa teve como objetivo verificar a força preditiva das doze categorias de necessidades educacionais especiais estudadas (variáveis independentes) sobre o repertório de habilidades sociais das crianças (variáveis dependentes), por meio da técnica estatística de Regressão Linear Múltipla (Tabachnick \& Fidell, 2007). Diferentemente da comparação entre grupos, a análise de Regressão Linear Múltipla permite verificar em que medida um conjunto de variáveis independentes pode predizer o comportamento de uma variável dependente. Nesse sentido, buscou-se testar a importância relativa das variáveis preditoras (necessidades especiais) na explicação da variável dependente (habilidades sociais), assim como verificar a magnitude do aumento da correlação múltipla resultante da subtração de variáveis independentes não significativas da equação (Abbad \& Torres, 2002).

\section{Método}

Este estudo foi aprovado pelo Comitê de Ética em Pesquisa da Universidade Federal de São Carlos (UFSCar; Protocolo 168/2008) e seguiu suas devidas recomendações. Os pais assinaram um termo de consentimento informado, autorizando a participação de seus filhos.

\section{Participantes}

Participaram 120 crianças com idades entre seis e 15 anos, estudantes de escolas regulares ou especiais, provenientes de quatro estados brasileiros (São Paulo, Minas Gerais, Paraná e Rio de Janeiro). Foram compostos 12 grupos, de 10 crianças cada, que atenderam aos critérios de indicadores para: (a) Autismo, (b) Deficiência Auditiva, (c) Deficiência Intelectual Leve, (d) Deficiência Intelectual Moderada, (e) Deficiência Visual, (f) Desvio Fonológico, (g) Dificuldades de Aprendizagem, (h) Dotação e Talento, (i) Problemas de Comportamento Externalizantes, (j) Problemas de Comportamento Internalizantes, (k) Problemas de Comportamento Internalizantes e Externalizantes e (1) Transtorno de Déficit de Atenção e Hiperatividade (TDAH). Nota-se que no presente estudo não foram abordadas todas as categorias de necessidades especiais descritas na lei IDEA (2004) e, além disso, foram utilizados alguns subgrupos específicos contidos dentro de uma mesma categoria, como no caso da Deficiência Intelectual (Leve e Moderada) e do Distúrbio Emocional (Problemas Externalizantes, Internalizantes e Misto). Para a inclusão de cada criança em determinado grupo, foram utilizados os critérios descritos abaixo.

Autismo. Diagnóstico prévio de Autismo primário $(N=5)$ ou Autismo associado a outros transtornos $(N=5)$, de acordo com os critérios do DSM-IV, CID-10 e Escala CARS (Schopler, Reichler, \& Renner, 1988). Os transtornos associados na amostra foram: Encefalopatia, Deficiência Intelectual Moderada, Síndrome do X Frágil, Paralisia Cerebral e Patologia de base primária inespecificada.

Deficiência Auditiva. Diagnóstico prévio de Deficiência Auditiva leve (de 25 a $40 \mathrm{db}$ ), moderada (de 41 a $55 \mathrm{db}$ ), acentuada (de 56 a $70 \mathrm{db}$ ), severa (de 71 a 90 $\mathrm{db}$ ) ou profunda (acima de $91 \mathrm{db}$ ), com base em exame audiométrico.

Deficiência Intelectual Leve. Diagnóstico prévio de Deficiência Intelectual Leve (quociente de inteligência [QI] de 50-55 a aproximadamente 70), com base em avaliação multidisciplinar do funcionamento intelectual e adaptativo, de acordo com os critérios do DSM-IV (APA, 2002).

Deficiência Intelectual Moderada. Diagnóstico prévio de Deficiência Intelectual Moderada (QI de 35-40 a 50-55), com base em avaliação multidisciplinar do funcionamento intelectual e adaptativo, de acordo com os critérios do DSM-IV (APA, 2002).

Deficiência Visual. Diagnóstico prévio de Baixa Visão (acuidade visual entre 6/60 e 18/60) ou Cegueira Total (acuidade visual de 6/60 ou menos), com base em exame oftalmológico, anamnese e avaliação funcional.

Desvio Fonológico. Diagnóstico prévio de Desvio Fonológico, com base no Teste de Linguagem Infantil nas Áreas de Fonologia, Vocabulário, Fluência e Pragmática (Andrade, Béfi-Lopes, Fernandes, \& Wertzner, 2004), que verifica os erros encontrados na fala da criança: omissões, substituições e distorções comuns e incomuns.

Dificuldades de Aprendizagem. Desempenho acadêmico inferior, segundo o resultado no Teste de Desempenho 
Freitas, L. C. \& Del Prette, Z. A. P. (2014). Categorias de Necessidades Educacionais Especiais Enquanto Preditoras de Déficits em Habilidades Sociais na Infância.

Escolar (TDE; Stein, 1994) e pontuação igual ou inferior ao percentil 10 na escala de competência acadêmica do Sistema de Avaliação de Habilidades Sociais -Versão Professor (SSRS-BR-Professor). Essas pontuações são baseadas nos padrões normativos do SSRS-BR, a partir de uma amostra de 955 crianças brasileiras (Bandeira, Del Prette, Del Prette, \& Magalhães, 2009).

Dotação e Talento. Identificação prévia de Dotação e Talento no domínio da Inteligência, com indicadores em pelo menos uma das seguintes áreas: (a) Inteligência e Capacidade Geral, (b) Talento Verbal e Pensamento Linear e/ou (c) Talento Científico-matemático e Pensamento Não-linear Abstrato. Essa identificação foi feita inicialmente por professores de escolas regulares por meio de observação direta, utilizando um instrumento composto por indicadores de Dotação e Talento (Guenther, 2006). As crianças identificadas passaram por processo de observação assistida em um centro especializado para Dotação e Talento, onde vivenciam situações de aprendizagem enriquecidas. Algumas crianças selecionadas para este estudo possuíam também indicadores em outros domínios de Dotação, incluindo as áreas de Criatividade, Talento Psicossocial e Talento Psicomotor.
Problemas de Comportamento Externalizantes. Crianças com pontuação igual ou superior ao percentil 90 na subescala de problemas de comportamento externalizantes do SSRS-BR-Professor e pontuação igual ou inferior ao percentil 50 na subescala de problemas de comportamento internalizantes do mesmo instrumento.

Problemas de Comportamento Internalizantes. Crianças com pontuação igual ou superior ao percentil 90 na subescala de problemas de comportamento internalizantes do SSRS-BR-Professor e pontuação igual ou inferior ao percentil 50 na subescala de problemas de comportamento externalizantes do mesmo instrumento.

Problemas de Comportamento Internalizantes e Externalizantes. Crianças com pontuação igual ou superior ao percentil 90 nas subescalas de problemas de comportamento externalizantes e internalizantes do SSRS-BR-Professor.

$T D A H$. Diagnóstico prévio de TDAH, com base em avaliação psicológica e acadêmica: observação direta na escola, teste WISC (Figueiredo, 2002), Escalas de Conners (Conners, Sitarenios, Parker, \& Epstein 1998) específica para TDAH e preenchimento dos critérios do DSM-IV (APA, 2002).

$\mathrm{Na}$ Tabela 1 são apresentadas as características sociodemográficas dos diferentes grupos em termos de sexo, idade, tipo de escola e estado brasileiro.

Tabela 1

Características Sociodemográficas dos Diferentes Grupos de Participantes

\begin{tabular}{|c|c|c|c|c|c|c|c|c|}
\hline \multirow{2}{*}{ Grupo } & \multicolumn{2}{|c|}{ Sexo* } & \multirow{2}{*}{$\begin{array}{l}\text { Idade } \\
M(D P)\end{array}$} & \multicolumn{4}{|c|}{ Tipo de escola\# } & \multirow{2}{*}{ Estado } \\
\hline & M & $\mathrm{F}$ & & Pub & Par & Esp & $\mathrm{Pub} / \mathrm{Esp}$ & \\
\hline Autismo & 6 & 4 & $11,30(2,06)$ & - & - & 10 & - & SP \\
\hline Deficiência Auditiva & 7 & 3 & $10,10(2,13)$ & 9 & 1 & - & - & SP e MG \\
\hline Deficiência Intelectual Leve & 6 & 4 & $11,80(1,23)$ & - & - & 10 & - & SP \\
\hline Deficiência Intelectual Moderada & 8 & 2 & $12,70(1,34)$ & - & - & 10 & - & SP \\
\hline Deficiência Visual & 6 & 4 & $8,50(1,35)$ & 9 & 1 & - & - & PR \\
\hline Desvio Fonológico & 7 & 3 & $7,40(0,70)$ & 9 & 1 & - & - & SP \\
\hline Dificuldades de Aprendizagem & 7 & 3 & $9,90(0,99)$ & 10 & - & - & - & SP \\
\hline Dotados e Talentosos & 4 & 6 & $10,60(1,26)$ & - & - & - & 10 & MG \\
\hline Problemas de Comportamento Externalizantes & 6 & 4 & $10,60(2,46)$ & 8 & 2 & - & - & SP, MG e RJ \\
\hline Problemas de Comportamento Internalizantes & 6 & 4 & $9,80(2,35)$ & 9 & 1 & - & - & MG e SP \\
\hline $\begin{array}{l}\text { Problemas de Comportamento Internalizantes } \\
\text { e Externalizantes }\end{array}$ & 5 & 5 & $7,30(1,64)$ & 6 & 4 & - & - & MG e SP \\
\hline TDAH & 10 & - & $11,30(1,57)$ & 9 & 1 & - & - & PR \\
\hline
\end{tabular}

Nota. *Sexo: M=Masculino, F=Feminino; \# Tipo de escola: Pub=Pública, Par=Particular, Esp=Especial, Pub/Esp= Pública e Especial.

\section{Instrumento de Avaliação}

Para a avaliação, foi utilizado o SSRS-BR-Professor. Este inventário, originalmente norte-americano (Social
Skills Rating System; Gresham \& Elliott, 1990), avalia as habilidades sociais, os problemas de comportamento e a competência acadêmica de crianças do ensino funda- 
mental, por meio de três questionários, respondidos por pais, estudantes e professores. O SSRS-BR se encontra validado para o Brasil para crianças com desenvolvimento típico (Bandeira et al., 2009) e deficiência intelectual (L. C. Freitas \& Del Prette, 2010a, 2010b), apresentando propriedades psicométricas adequadas de validade de construto, consistência interna, validade de critério e estabilidade temporal. Na presente pesquisa, foi utilizada somente a versão para professores, com 30 itens que avaliam a frequência e a importância das habilidades sociais das crianças, 18 itens que avaliam a frequência de problemas de comportamento e nove itens que avaliam a competência acadêmica dos estudantes. Os itens são respondidos em uma escala de frequência, que varia de 0 a $2(0=$ nunca, $1=$ algumas vezes e $2=$ muito frequente $) \mathrm{e}$ uma escala de importância, que também varia de 0 a $2(0=$ não importante, $1=$ importante e $2=$ muito importante). A escala de competência acadêmica possui cinco alternativas de respostas, que visam classificar os alunos com relação à sua turma: $1=10 \%$ piores, $2=20 \%$ piores, $3=40 \%$ médios, $4=20 \%$ bons e $5=10 \%$ ótimos. A análise fatorial da versão para professores indicou cinco subescalas de habilidades sociais (responsabilidade/cooperação, asserção positiva, autocontrole, autodefesa e cooperação com pares), duas de problemas de comportamento (problemas internalizantes e problemas externalizantes) e uma única para competência acadêmica (Bandeira et al., 2009).

\section{Procedimento de Coleta de Dados}

Desde o inicio do processo de validação do SSRS-BR para o Brasil, vários estudos conduzidos por integrantes do grupo de pesquisa responsável por sua adaptação nacional, vêm utilizando esse instrumento com objetivos diversos. O presente estudo reuniu dados coletados pelas pesquisas desse conjunto que utilizaram o professor como avaliador do repertório social e acadêmico das crianças (Bandeira et al., 2009; Barreto et al., 2011; Carlino, 2010; L C. Freitas \& Del Prette, 2010a; M. G. Freitas, 2005; M. L. P. F. Freitas, 2012; Rocha, 2009; Souza, 2008). A coleta de dados realizada com os professores, em todos os estudos, seguiu um procedimento geral padronizado, elaborado com base nas instruções de aplicação do instrumento. Foram realizados breves encontros com os professores, para fornecer as instruções de preenchimento dos instrumentos e esclarecer as suas dúvidas. Após esse primeiro momento, os professores preenchiam os instrumentos com o auxílio do aplicador, em horários livres na própria escola ou sozinhos em casa, conforme sua disponibilidade. Os professores responderam um instrumento para cada aluno de sua classe que participou da pesquisa e nos casos em que os professores preencheram os questionários sozinhos, os mesmos foram devolvidos ao pesquisador em um intervalo médio de 15 a 20 dias.

\section{Análise e Tratamento dos Dados}

Os dados coletados ou reunidos para este estudo foram digitados em uma planilha do programa SPSS-PC
(Statistical Package for Social Sciences), versão 17.0. Inicialmente, foi verificada a normalidade do escore global de habilidades sociais na amostra (teste de Kolmogorov-Smirnov) e a consistência interna do SSRS-BR-Professor (Alfa de Cronbach), bem como a possível influência da idade (correlação Pearson com o escore de habilidades sociais) e do sexo (Teste $t$ de student) sobre o repertório de habilidades sociais da amostra, a fim de se controlar vieses relacionados a essas variáveis. Para verificar o grau de predição de cada categoria de necessidades educacionais especiais sobre o repertório de habilidades sociais das crianças, foram realizadas Análises de Regressão Linear Múltipla, tendo como variáveis dependentes os escores global e das subescalas de habilidades sociais (Tabachnick \& Fidell, 2007). Em todos os testes inferenciais realizados foi adotado como nível de significância um valor de, pelo menos, $p<0,05$.

Análises Preliminares. Os testes efetuados mostraram a normalidade da distribuição do escore global de habilidades sociais $(z=0,94, p=0,33)$ e das subescalas de Responsabilidade/Cooperação ( $z=1,06, p=0,21)$, Asserção Positiva $(z=0,96, p=0,32)$, Autocontrole $(z=0,76, p=0,61)$, Autodefesa $(z=1,33, p=0,06)$ e Cooperação com Pares $(z=1,31, p=0,06)$, possibilitando a utilização de testes estatísticos paramétricos nesta amostra. A consistência interna da escala global e subescalas de habilidades sociais indicou índices altos do Alfa de Cronbach acima de 0,90 para a escala global e acima de 0,70 para todos os fatores: Responsabilidade/Cooperação $(\alpha=0,94)$, Asserção Positiva $(\alpha=0,86)$, Autocontrole $(\alpha=0,86)$, Autodefesa $(\alpha=0,76)$ e Cooperação com Pares $(\alpha=0,71)$.

A variável idade não se correlacionou de maneira significativa com o escore global e subescalas de habilidades sociais. Com relação ao sexo, não foram encontradas diferenças significativas entre meninos e meninas no escore global de habilidades sociais e subescalas.

Análises de Regressão. Em cada modelo de regressão foram incluídas, inicialmente, por meio do método Enter, todas as categorias de necessidades especiais enquanto variáveis independentes, entendidas como preditoras da variação nas habilidades sociais (variável dependente). Em um segundo momento, foram retiradas as variáveis independentes (categorias de necessidades especiais) que não se associaram de maneira significativa com o modelo, restando apenas as variáveis com contribuição significativa. Nos modelos testados, as variáveis independentes (necessidades especiais) foram consideradas enquanto variáveis categóricas (presença ou ausência), sendo que cada criança da amostra apresentava apenas uma das 12 necessidades especiais estudadas.

Verificou-se ainda o grau de colinearidade entre as variáveis preditoras do modelo, ou seja, apenas as variáveis independentes que não apresentavam associações significativas entre si foram mantidas no modelo (VIF $<10$; Hosmer \& Lemeshow, 2000). Em todos os modelos de regressão executados não foi observado o problema da multicolinearidade. 
Freitas, L. C. \& Del Prette, Z. A. P. (2014). Categorias de Necessidades Educacionais Especiais Enquanto Preditoras de Déficits em Habilidades Sociais na Infância.

Considerando-se que foram testados vários modelos de regressão, foi utilizada a correção de Bonferroni para a análise e interpretação dos resultados (Abdi, 2007). A realização de uma série de análises de regressão aumenta as chances de ocorrer erros do Tipo I, ou seja, associações não significativas dentro do intervalo de confiança inicialmente estipulado $(p<0,05)$. A correção de Bonferroni altera o nível de significância a fim de evitar erros derivados das múltiplas análises. Como foram testados seis modelos de regressão (para a escala global e para as cinco subescalas), duas vezes cada (um com todas as necessidades especiais e um segundo incluindo apenas as significativas), dividiu-se o valor de $p$ pelo número de testes realizados $(0,05 / 12$; Abdi, 2007). Sendo assim, o valor de $p$ com a correção de Bonferroni foi de 0,004 .

\section{Resultados}

Os dados obtidos nas análises de regressão, para o escore geral de habilidades sociais e para cada uma das subescalas do SSRS-BR, são apresentados a seguir.

\section{Escore Global de Habilidades Sociais}

Na primeira análise de regressão realizada para o escore global de habilidades sociais, foram inseridas conjuntamente todas as 12 categorias de alunos com necessidades especiais enquanto variáveis preditoras. Em seu conjunto, as categorias de necessidades especiais explicaram $28 \%$ da variância $\left(R^{2}\right)$ no escore global de habilidades sociais, sendo esse modelo estatisticamente significativo $[F$ $(119)=3,74 ; p<0,001]$.

Entretanto, apenas cinco necessidades especiais contribuíram significativamente para a variação no escore global de habilidades sociais: TDAH, Problemas de Comportamento Internalizantes e Externalizantes, Autismo, Problemas de Comportamento Externalizantes e Dificuldades de Aprendizagem. Por esse motivo, uma nova análise de regressão foi realizada, retirando-se as variáveis não significativas e mantendo-se apenas as cinco mencionadas. A Tabela 2 apresenta os resultados das análises finais de regressão múltipla para o escore global de habilidades sociais e subescalas.

Tabela 2

Análise de Regressão Linear Múltipla para a Predição do Escore Global de Habilidades Sociais e Subescalas

\begin{tabular}{|c|c|c|c|c|c|c|}
\hline Variáveis Dependentes & Variáveis Preditoras & B & $t$ & $\beta$ & $p$ & $R^{2}$ \\
\hline \multirow{5}{*}{$\begin{array}{l}\text { Escore Global de Habilidades } \\
\text { Sociais }\end{array}$} & TDAH & $-15,69$ & $-4,03$ & $-0,34$ & $<\mathbf{0 , 0 0 1 *}$ & 0,065 \\
\hline & Problemas Internalizantes e Externalizantes & $-12,79$ & $-3,29$ & $-0,28$ & $<0,001 *$ & 0,045 \\
\hline & Autismo & $-11,59$ & $-2,98$ & $-0,25$ & $<0,001 *$ & 0,042 \\
\hline & Problemas Externalizantes & $-9,89$ & $-2,54$ & $-0,22$ & 0,01 & 0,035 \\
\hline & Dificuldades de Aprendizagem & $-8,79$ & $-2,26$ & $-0,19$ & 0,03 & 0,035 \\
\hline \multirow{3}{*}{$\begin{array}{l}\text { Subescala de Responsabilidade/ } \\
\text { Cooperação }\end{array}$} & TDAH & $-8,34$ & $-3,53$ & $-0,30$ & $<0,001 *$ & 0,063 \\
\hline & Problemas Internalizantes e Externalizantes & $-7,04$ & $-2,99$ & $-0,25$ & $<0,001 *$ & 0,063 \\
\hline & Problemas Externalizantes & $-7,44$ & $-3,15$ & $-0,27$ & $<0,001 *$ & 0,058 \\
\hline \multirow[t]{3}{*}{ Subescala de Asserção Positiva } & Autismo & $-4,28$ & $-3,25$ & $-0,28$ & $<0,001 *$ & 0,065 \\
\hline & Problemas Internalizantes & $-4,58$ & $-3,47$ & $-0,30$ & $<\mathbf{0 , 0 0 1 *}$ & 0,063 \\
\hline & TDAH & $-3,28$ & $-2,49$ & $-0,21$ & 0,01 & 0,044 \\
\hline \multirow[t]{4}{*}{ Subescala de Autocontrole } & Problemas Externalizantes & $-5,65$ & $-4,40$ & $-0,35$ & $<0,001 *$ & 0,076 \\
\hline & Problemas Internalizantes e Externalizantes & $-5,15$ & $-4,01$ & $-0,32$ & $<0,001 *$ & 0,072 \\
\hline & TDAH & $-4,65$ & $-3,62$ & $-0,29$ & $<0,001 *$ & 0,069 \\
\hline & Autismo & $-3,95$ & $-3,08$ & $-0,25$ & $<\mathbf{0 , 0 0 1 *}$ & 0,060 \\
\hline \multirow[t]{6}{*}{ Subescala de Autodefesa } & Dificuldades de Aprendizagem & $-2,18$ & $-4,08$ & $-0,34$ & $<0,001 *$ & 0,061 \\
\hline & Autismo & $-2,28$ & $-4,27$ & $-0,36$ & $<0,001 *$ & 0,058 \\
\hline & Problemas Internalizantes & $-1,78$ & $-3,33$ & $-0,28$ & $<0,001 *$ & 0,042 \\
\hline & TDAH & $-1,58$ & $-2,96$ & $-0,25$ & $<0,001 *$ & 0,038 \\
\hline & Deficiência Intelectual Moderada & $-1,28$ & $-2,40$ & $-0,20$ & 0,02 & 0,038 \\
\hline & Desvio Fonológico & $-1,28$ & $-2,40$ & $-0,20$ & 0,02 & 0,028 \\
\hline \multirow{2}{*}{$\begin{array}{l}\text { Subescala de Cooperação com } \\
\text { Pares }\end{array}$} & TDAH & $-1,78$ & $-2,63$ & $-0,23$ & 0,01 & 0,047 \\
\hline & Problemas Externalizantes & $-1,38$ & $-2,04$ & $-0,18$ & 0,04 & 0,033 \\
\hline
\end{tabular}

* $p<0,004$, com correção de Bonferroni. 
O conjunto dessas cinco categorias de necessidades especiais explicou $22 \%$ da variância $\left(R^{2}\right)$ no escore global de habilidades sociais, em sentido negativo $[F(119)=6,52$; $p<0,001]$. Os coeficientes de regressão padronizados $(\beta)$ indicam a proporção em que as mudanças nas unidades de desvio-padrão da variável preditiva afetam as mudanças no desvio-padrão da variável dependente. De acordo com Floyd, Evans e McGrew (2003), os coeficientes de regressão padronizados variando de 0,10 a 0,29 sinalizam relações moderadas entre as variáveis, enquanto coeficientes acima de 0,30 apontam fortes relações entre as variáveis.

Nesse modelo, a categoria que melhor explicou a variação nas habilidades sociais foi o TDAH, responsável por uma predição forte negativa $(\beta=-0,34)$ de aproximadamente $6,5 \%(p<0,001)$. Em seguida, as categorias de necessidades especiais com maior poder explicativo foram Problemas de Comportamento Internalizantes e Externalizantes $(4,5 \% ; p<0,001)$ e Autismo $(4 \% ; p<0,001)$, ambas consideradas relações negativas moderadas ( $\beta$ entre $-0,19$ e -0,28). Considerando-se a aplicação da correção de Bonferroni, o peso individual das variáveis Problemas Externalizantes $(3,5 \% ; p=0,01)$ e Dificuldades de Aprendizagem $(3,5 \% ; p=0,03)$ na equação não foi estatisticamente significativo.

\section{Subescala de Responsabilidade/Cooperação}

$\mathrm{Na}$ primeira análise de regressão para a subescala de Responsabilidade/Cooperação, as 12 categorias de alunos com necessidades especiais, em seu conjunto, explicaram $28 \%$ da variância, sendo esse modelo estatisticamente significativo $[F(119)=3,74 ; p<0,001]$. Como apenas três necessidades especiais contribuíram significativamente para a variação nessa subescala, uma nova análise de regressão foi realizada, mantendo-se apenas as três significativas: TDAH, Problemas de Comportamento Externalizantes e Problemas de Comportamento Internalizantes e Externalizantes.

O conjunto dessas três categorias explicou $18 \%$ da variância $\left(R^{2}\right)$ do escore de Responsabilidade/Cooperação, em sentido negativo $[F(119)=8,70 ; p<0,001]$. A categoria que melhor explicou a variação em Responsabilidade/Cooperação foi o TDAH, responsável por uma predição forte negativa $(\beta=-0,30)$ de aproximadamente $6 \%$. Em seguida, as categorias com maior poder explicativo foram Problemas de Comportamento Internalizantes e Externalizantes e Problemas de Comportamento Externalizantes, ambas com $6 \%$ de predição, porém com associações negativas consideradas moderadas ( $\beta$ entre $-0,25$ e $-0,27$ ).

\section{Subescala de Asserção Positiva}

$\mathrm{Na}$ primeira análise de regressão para a subescala de Asserção Positiva, as 12 categorias de necessidades especiais explicaram $28 \%$ da variância, sendo esse modelo estatisticamente significativo [ $F(119)=3,81 ; p<0,001]$. Como apenas três categorias contribuíram significativamente para a variação nessa subescala, uma nova análise de regressão foi realizada, mantendo-se apenas as três significativas: TDAH, Autismo e Problemas de Comportamento Internalizantes.

O conjunto dessas três categorias explicou $17 \%$ da variância $\left(R^{2}\right)$ do escore de Asserção Positiva, em sentido negativo $[F(119)=8,05 ; p<0,001]$. A categoria que melhor explicou a variação em Asserção Positiva foi o Autismo, responsável por uma predição moderada negativa $(\beta=-$ $0,28)$ de aproximadamente $(7 \% ; p<0,001)$. Em seguida, a categoria com maior poder explicativo foi Problemas de Comportamento Internalizantes $(6 \% ; p<0,001)$, com associação negativa forte $(\beta=-0,30)$. Considerando-se a aplicação da correção de Bonferroni, o peso individual da variável TDAH na equação $(4 \%, p=0,01)$ não foi estatisticamente significativo.

\section{Subescala de Autocontrole}

Na primeira análise de regressão para a subescala de Autocontrole, as 12 categorias de necessidades especiais explicaram $34 \%$ da variância, sendo esse modelo estatisticamente significativo $[F(119)=5,09 ; p<0,001]$. Como apenas quatro categorias contribuíram significativamente para a variação nessa subescala, uma nova análise de regressão foi realizada, mantendo-se apenas as quatro significativas: TDAH, Autismo, Problemas de Comportamento Externalizantes e Problemas de Comportamento Internalizantes e Externalizantes.

O conjunto dessas quatro categorias explicou $28 \%$ da variância $\left(R^{2}\right)$ do escore de Autocontrole, em sentido negativo $[F(119)=10,99 ; p<0,001]$. A categoria que melhor explicou a variação em Autocontrole foi Problemas de Comportamento Externalizantes, responsável por uma predição forte negativa $(\beta=-0,35)$ de aproximadamente $8 \%$. Em seguida, as categorias com maior poder explicativo foram: Problemas de Comportamento Internalizantes e Externalizantes $(7 \%)$, em associação negativa forte $(\beta=-$ $0,32)$, TDAH (7\%) e Autismo (6\%), as duas últimas com relações negativas moderadas ( $\beta$ entre $-0,25$ e $-0,29$ ).

\section{Subescala de Autodefesa}

Na primeira análise de regressão para a subescala de Autodefesa, as 12 categorias de necessidades especiais explicaram $30 \%$ da variância, sendo esse modelo estatisticamente significativo $[F(119)=4,21 ; p<0,001]$. Como apenas seis categorias contribuíram significativamente para a variação nessa subescala, uma nova análise de regressão foi realizada, mantendo-se apenas as seis significativas: Deficiência Intelectual Moderada, TDAH, Autismo, Desvio Fonológico, Problemas de Comportamento Internalizantes e Dificuldades de Aprendizagem.

O conjunto dessas seis categorias explicou $26 \%$ da variância $\left(R^{2}\right)$ do escore de Autodefesa, em sentido negativo $[F(119)=6,77 ; p<0,001]$. A categoria que melhor explicou a variação em Autodefesa foi Dificuldades de Aprendizagem, responsável por uma predição forte negativa $(\beta=-0,34)$ de aproximadamente $6 \%(p<0,001)$. Em seguida, as categorias com maior poder explicativo foram: Autismo $(6 \% ; p<0,001)$, em associação negativa forte 
Freitas, L. C. \& Del Prette, Z. A. P. (2014). Categorias de Necessidades Educacionais Especiais Enquanto Preditoras de Déficits em Habilidades Sociais na Infância.

$(\beta=-0,36)$, Problemas de Comportamento Internalizantes $(4 \% ; p<0,001)$ e TDAH $(4 \% ; p<0,001)$, as duas últimas com relações negativas moderadas ( $\beta$ entre $-0,20$ e $-0,28)$. Considerando-se a aplicação da correção de Bonferroni, o peso individual das variáveis Deficiência Intelectual Moderada $(4 \% ; p=0,02)$ e Desvio Fonológico $(3 \% ; p=0,02)$ na equação não foi estatisticamente significativo.

\section{Subescala de Cooperação com Pares}

$\mathrm{Na}$ primeira análise de regressão para a subescala de Cooperação com Pares, as 12 categorias de necessidades especiais explicaram $18 \%$ da variância $[F(119)=2,16$; $p=0,02]$. Como apenas algumas categorias contribuíram significativamente para a variação nessa subescala, outras três análises de regressão foram realizadas, mantendo-se apenas as significativas. $\mathrm{O}$ modelo final para a subescala de Cooperação com Pares foi composto apenas pelo TDAH e Problemas de Comportamento Externalizantes. Essas duas categorias juntas explicaram $8 \%$ da variância $\left(R^{2}\right)$ do escore de Cooperação com Pares, em sentido negativo $[F(119)=5,08 ; p=0,01]$. Entretanto, considerando-se a aplicação da correção de Bonferroni, o modelo encontrado não foi estatisticamente significativo $(p=0,01)$.

Cabe ressaltar que, das 12 categorias de necessidades especiais, seis não se constituíram como variável preditora significativa em nenhum dos modelos de regressão: Desvio Fonológico, Dotação e Talento, Deficiência Visual, Deficiência Intelectual Leve, Deficiência Intelectual Moderada e Deficiência Auditiva.

\section{Discussão}

As análises de regressão permitiram identificar os conjuntos de categorias de necessidades educacionais especiais melhores preditores da variância no escore global e subescalas de habilidades sociais. Em todos os modelos, foram mantidas apenas as categorias que apresentaram contribuição preditiva significativa. Deve-se destacar ainda, que os coeficientes de regressão padronizados $(\beta)$ foram negativos em todos os modelos finais testados, indicando que o aumento nas unidades das variáveis independentes (necessidades especiais) correspondeu a diminuições nos valores das variáveis dependentes (habilidades sociais) (Floyd et al., 2003). Nesse sentido, pode-se dizer que os modelos finais indicaram as categorias de alunos com necessidades especiais como variáveis preditoras de déficits em habilidades sociais.

Os resultados apontaram que a combinação de quatro categorias de necessidades especiais - TDAH, Problemas de Comportamento Externalizantes, Mistos (Internalizantes e Externalizantes) e Autismo - constituem preditores mais fortes de déficits para o escore global de habilidades sociais gerais e para as subescalas de Responsabilidade/ Cooperação e Autocontrole. Pode-se considerar que as crianças enquadradas nessas categorias de necessidades especiais apresentam em seu repertório uma série de comportamentos problemáticos incompatíveis com o desempenho de habilidades sociais (Hanley, Iwata, \& McCord, 2003), sendo essa uma possível hipótese explicativa para o fato de serem as principais preditoras de déficits em habilidades sociais (Del Prette \& Del Prette, 2005a; Gresham, 2009).

No caso específico do TDAH, que apareceu como variável preditora em todos os modelos e como principal preditora para o escore global de habilidades sociais, supõe-se que as dificuldades dessas crianças em regular a atenção e a impulsividade contribuem enormemente para os déficits em comportamentos prossociais, conforme hipotetizaram Hay, Hudson e Liang (2010). Além das características próprias do TDAH, deve-se salientar que essas crianças podem apresentar alta frequência de problemas de comportamento (Marton, Wiener, Rogers, Moore, \& Tannock, 2009) e ser frequentemente rejeitadas por seus pares (Mrug et al., 2009), fatores esses que influenciam negativamente o repertório de habilidades sociais na infância.

A deficiência em autocontrole também pode explicar, em parte, os déficits em habilidades sociais em crianças com TDAH. Os experimentos de Hoerger e Mace (2006) e Neef et al. (2005) ilustraram o modelo comportamental de autocontrole de Barkley (1997), no qual se prevê que o comportamento das crianças com TDAH tem maior probabilidade de ser afetado por consequências imediatas do que por consequências atrasadas. Para o cumprimento de tarefas acadêmicas, as crianças com TDAH apresentaram mais frequentemente o padrão de escolher pequenas recompensas imediatas do que uma quantidade maior de recompensa com atraso, comparadas às crianças sem TDAH (Hoerger \& Mace, 2006; Neef et al., 2005). Segundo os autores, esse padrão é considerado impulsivo porque uma maior quantidade de reforço é perdida em favor de uma menor quantidade imediata (Hoerger \& Mace, 2006; Neef et al., 2005). Transpondo-se esse modelo para o comportamento social, pode-se supor que as respostas que produzem reforçamento mais imediato (por exemplo, os comportamentos problemáticos) são emitidas mais frequentemente pelas crianças com TDAH, que perdem, com isso, importantes oportunidades de aprendizagem de comportamentos alternativos como as diferentes classes de habilidades sociais, deixando de responder a determinadas pistas sociais antecedentes para se comportar de forma apropriada em sala de aula.

Os resultados referentes aos Problemas de Comportamento Misto (Internalizantes e Externalizantes) sugerem que a combinação desses dois tipos de problemas constitui um agravante para déficits em habilidades sociais. De acordo com Gresham (2009), os problemas de comportamentos são concorrentes do comportamento socialmente habilidoso, podendo bloquear a aquisição e o desempenho de determinadas habilidades sociais. Esse efeito pode ocorrer se a criança apresenta apenas problemas do tipo internalizante ou externalizante, mas os dados deste estudo permitiram verificar que a combinação de ambos resulta em déficits ainda maiores em habilidades sociais.

Os modelos de regressão para as subescalas de Asserção Positiva e Autodefesa apresentaram uma configuração 
ligeiramente diferente daqueles até aqui considerados, com destaque para três variáveis: Autismo, Problemas Internalizantes e Dificuldades de Aprendizagem. Para a subescala de Asserção Positiva, o Autismo apareceu ao lado dos Problemas Internalizantes como preditor significativo. Para a subescala de Autodefesa, as Dificuldades de Aprendizagem apareceram enquanto principal preditor ao lado do Autismo, Problemas Internalizantes e TDAH.

Aqui se pode notar o papel do Autismo na predição de déficits em habilidades sociais, em particular as mais complexas como as de civilidade, comunicação e expressão de sentimentos (Del Prette \& Del Prette, 2005a). Considerando que o prejuízo acentuado na interação social constitui uma das características centrais do Autismo (APA, 2002; Macintosh \& Dissanayake, 2006), pode-se inferir que as habilidades sociais que necessitam de requisitos comportamentais mais elaborados, como é o caso da Asserção e Autodefesa, sejam ainda mais deficitárias no repertório dessas crianças. A depender da severidade do transtorno, é possível até mesmo que essas habilidades nunca tenham sido aprendidas, configurando-se como déficits de aquisição (Del Prette \& Del Prette, 2005a; Gresham, 2009).

Os Problemas de Comportamento Internalizantes, caracterizados principalmente por indicadores de ansiedade, depressão e isolamento, também foram destaque enquanto preditores de déficits em Asserção Positiva e Autodefesa. Essas habilidades sociais, por definição, envolvem algum risco potencial de reação indesejável por parte do interlocutor, podendo implicar maior dificuldade, ansiedade ou custo subjetivo a essas crianças (Del Prette \& Del Prette, 2005b). Assim, fugir ou esquivar-se dessas situações pode ser um comportamento reforçado negativamente pela diminuição da ansiedade ou das cognições negativas associadas ao próprio desempenho (Del Prette \& Del Prette, 2009; Rubin et al., 2009). Ao se envolverem nesse processo de fuga/esquiva, as crianças com Problemas Internalizantes estão perdendo oportunidades de aprendizagem e desempenho de habilidades sociais (Bornstein, Hahn, \& Haynes, 2010; Hymel, Rubin, Rowden, \& LeMare, 1990), especialmente em situações potencionalmente mais difíceis e ansiogênicas.

Para a subescala de Autodefesa, as Dificuldades de Aprendizagem apresentaram a maior força preditiva de déficits em comparação com as todas as outras variáveis do modelo. Essa categoria apareceu, ainda, como última preditora de déficits no escore global de habilidades sociais. Apesar de já existir uma extensa literatura que relaciona dificuldades acadêmicas a prejuízos em habilidades sociais (Caprara et al., 2000; Feitosa et al., 2012), esse resultado adiciona um elemento novo, apontando uma classe de habilidades que parece ser criticamente deficitária nesse grupo de crianças. Como uma implicação desse achado, os programas de intervenção devem procurar ir além do ensino das habilidades sociais acadêmicas, já reconhecidas como deficitárias nessa população (Fumo, 2009), e ensinar formas socialmente adequadas de expressar ideias e opiniões e defender-se em situações consideradas injustas.

\section{Considerações Finais}

As categorias de necessidades especiais que foram as melhores preditoras de déficits nas análises de regressão realizadas neste estudo foram, consistentemente, as que apresentaram menores frequências de habilidades sociais no estudo de L. C. Freitas e Del Prette (2013), ao realizarem o teste de comparações múltiplas ANOVA: TDAH, Autismo, Problemas de Comportamento Misto, Problemas Externalizantes, Problemas Internalizantes e Dificuldades de Aprendizagem. A diferença, neste estudo, foi o levantamento da possibilidade de se fazer predições do efeito dessas variáveis sobre as habilidades sociais. O conhecimento detalhado das características do repertório social de crianças com diferentes necessidades especiais, disponibilizado por esses dois estudos, torna-se potencialmente útil para o levantamento de grupos que devem receber assistência prioritária em programas de intervenção. Nesse ponto, a atuação conjunta de psicólogos, pais e professores pode ser imprescindível para prover condições que otimizem um desenvolvimento socioemocional saudável a esses grupos de crianças, uma vez que esses comportamentos são socialmente relevantes e empiricamente relacionados a indicadores de bom ajustamento psicológico e escolar (Berry \& O'Connor, 2009; Cia \& Barham, 2009).

Os resultados desta pesquisa sugerem que os problemas de comportamento, principalmente os externalizantes e disruptivos, podem desempenhar um papel importante na relação entre necessidades especiais e déficits em habilidades sociais. Seria interessante, portanto, que novas pesquisas investigassem a força e a possível direção da relação entre essas três variáveis (necessidades especiais, problemas de comportamento e habilidades sociais), por exemplo, investigando-as sob um modelo de variáveis moderadoras e mediadoras, conforme proposto por Baron e Kenny (1986).

Como limitações metodológicas, que poderiam ser aprimoradas em estudos futuros, encontram-se o número limitado de crianças em cada categoria de necessidades especiais $(N=10)$ e a diferença de critérios no processo de identificação e seleção de cada grupo, que envolveram: o resultado em escalas de avaliação, observação direta por professores, testes de desempenho escolar e de inteligência, avaliações psiquiátricas de acordo com manuais reconhecidos da área e exames médicos específicos, como o audiométrico e o oftalmológico. Além disso, alguns grupos do estudo já possuíam um diagnóstico/avaliação prévios enquanto outros foram selecionados segundo uma avaliação do próprio professor, como no caso das crianças com dificuldades de aprendizagem e problemas de comportamento.

Deve-se esclarecer, ainda, que as crianças não passaram por outras avaliações que permitissem descartar completamente outras deficiências ou transtornos associados. Exemplificando, as crianças com dificuldades de aprendizagem não foram submetidas a baterias de testes de inteligência ou a consultas psiquiátricas a fim de se eli- 
Freitas, L. C. \& Del Prette, Z. A. P. (2014). Categorias de Necessidades Educacionais Especiais Enquanto Preditoras de Déficits em Habilidades Sociais na Infância.

minar eventuais suspeitas sobre a existência de deficiência intelectual ou transtorno correlato. Sendo assim, pode-se supor que a eliminação dos vieses de seleção só ocorreria caso todas as crianças passassem por uma ampla avaliação que envolvesse todas as áreas do desenvolvimento infantil, utilizando-se os mesmos procedimentos e instrumentos e com a participação de diferentes profissionais da área de saúde e educação. Reconhecendo que uma avaliação deste nível seria extremamente desejável, porém apresenta altos custos em termos financeiros, de tempo e de recursos humanos, admite-se esse ponto como uma limitação da validade interna do estudo. Em pesquisas futuras, a especial atenção a esses fatores pode produzir achados mais confiáveis sobre o grau de predição das necessidades especiais sobre os déficits em habilidades sociais.

Um ponto final a ser destacado é que, um estudo de regressão, como no presente caso, é o retrato de uma situação que envolve, necessariamente, a interação entre as limitações das crianças com necessidades especiais e a forma como o ambiente tem lidado com elas. Em outras palavras, não se está advogando que, necessariamente, tais crianças terão déficits de habilidades sociais, mas, considerando que as habilidades sociais são aprendidas e que dependem do contexto situacional cultural, conforme destacam Del Prette e Del Prette (2010/2012), se o ambiente delas não se modificar, a tendência será um repertório mais empobrecido de habilidades sociais e, certamente, mais problemas de comportamento. Daí a importância de um estudo que permite prever, e, portanto, prevenir esses desdobramentos.

\section{Referências}

Abbad, G., \& Torres, C. V. (2002). Regressão múltipla stepwise e hierárquica em Psicologia Organizacional: Aplicações, problemas e soluções [Edição especial]. Estudos de Psicologia (Natal), 7, 19-29. doi:10.1590/S1413-294X2002000300004

Abdi, H. (2007). Bonferroni and Sidák corrections for multiple comparisons. In N. J. Salkind (Ed.), Encyclopia of measurement and statistics (pp. 103-107). Thousand Oaks, CA: Sage.

American Psychiatric Association. (2002). Manual diagnóstico e estatístico de transtornos mentais (DSM-IV-TR). São Paulo, SP: Artmed.

Andrade, C. R. F., Béfi-Lopes, D. M., Fernandes, F. D. M., \& Wertzner, H. F. (2004). ABFW: Teste de linguagem infantil nas áreas de fonologia, vocabulário, fluência e pragmática. Barueri: Pró-Fono.

Angélico, A. P., \& Del Prette, A. (2011). Avaliação do repertório de habilidades sociais de adolescentes com síndrome de Down. Psicologia: Reflexão e Crítica, 24(2), 207-217. doi:10.1590/S0102-79722011000200001

Bain, S. K., \& Bell, S. M. (2004). Social self-concept, social attributions, and peer relationships in fourth, fifth, and sixth graders who are gifted compared to high achievers. Gifted Child Quarterly, 48(3), 168-178. doi:10.1177/001698620404800302

Bandeira, M., Del Prette, Z. A. P., Del Prette, A., \& Magalhães, T. (2009). Validação das Escalas de Habilidades Sociais, Comportamentos Problemáticos e Competência Acadêmica (SSRS-BR) no ensino fundamental. Psicologia: Teoria e Pesquisa, 25(2), 271-282. doi:10.1590/S010237722009000200016
Bandeira, M., Rocha, S. S., Pires, L. G., Del Prette, Z. A. P., \& Del Prette, A. (2006). Competência acadêmica de crianças do ensino fundamental: Características sociodemográficas e relação com habilidades sociais. Interação, 10(1), 53-62.

Barkley, R. A. (1997). Behavioral inhibition, sustained attention, and executive functions: Constructing a unifying theory of ADHD. Psychological Bulletin, 121(1), 65-94.

Baron, R. M., \& Kenny, D. A. (1986). The moderator-mediator variable distinction in social psychological research: Conceptual, strategic, and statistical considerations. Journal of Personality and Social Psychology, 51(6), 1173-1182. doi:10.1037/0022-3514.51.6.1173

Barreto, S. O., Freitas, L. C., \& Del Prette, Z. A. P. (2011). Habilidades sociais na comorbidade entre dificuldade de aprendizagem e problemas de comportamento: Uma avaliação multimodal. Psico (Porto Alegre), 42(4), 503-510.

Berry, D., \& O'Connor, E. (2009). Behavioral risk, teacher-child relationships, and social skill development across middle childhood: A child-by-environment analysis of change. Journal of Applied Developmental Psychology, 31(1), 1-14. doi:10.1016/j.appdev.2009.05.001

Bornstein, M. H., Hahn, C. S., \& Haynes, O. M. (2010). Social competence, externalizing, and internalizing behavioral adjustment from early childhood through early adolescence: Developmental cascades. Development and Psychopathology, 22, 717-735. doi:10.1017/S0954579410000416

Caprara, V. G., Barbaranelli, C., Pastorelli, C., Bandura, A., \& Zimbardo, P. G. (2000). Prosocial foundations of children's academic achievement. Psychological Science, 11(4), 302306.

Carlino, F. C. (2010). Relação entre inteligibilidade de fala e habilidades sociais de comunicação em crianças com desvio fonológico (Dissertação de mestrado, Programa de Pós-Graduação em Educação Especial, Universidade Federal de São Carlos, SP, Brasil).

Cia, F., \& Barham, E. J. (2009). O envolvimento paterno e o desenvolvimento social de crianças iniciando as atividades escolares. Psicologia em Estudo, 14(1), 67-74.

Conners, C. K., Sitarenios, G., Parker, J. D. A., \& Epstein, J. N. (1998). The Revised Conners' Parent Rating Scale (CPRS-R): Factor structure, reliability, and criterion validity. Journal of Abnormal Child Psychology, 26(4), 257-268.

Costa, C. S. L., \& Del Prette, A. (2012). Estudo comparativo de observação de habilidades sociais de gêmeas com e sem deficiência visual. Revista Educação Especial, 25(42), 75-88. doi:10.5902/1984686X3552

Del Prette, Z. A. P., \& Del Prette, A. (2005a). Psicologia das habilidades sociais na infância: Teoria e prática. Petrópolis, RJ: Vozes.

Del Prette, Z. A. P., \& Del Prette, A. (2005b). Sistema multimídia de habilidades sociais de crianças (SMHSC-Del Prette). Manual. São Paulo, SP: Casa do Psicólogo.

Del Prette, Z. A. P., \& Del Prette, A. (2009). Psicologia das habilidades sociais: Terapia e educação. Petrópolis, RJ: Vozes.

Del Prette, Z. A. P., \& Del Prette, A. (2012). Social skills and behavior analysis: Historical proximity and new issues. Perspectivas em Análise do Comportamento, 1(2), 104-115. (Original work published 2010)

Del Prette, Z. A. P., Del Prette, A., Oliveira, L. A., Gresham, F. M., \& Vance, M. J. (2012). Role of social performance in predicting learning problems: Prediction of risk using logistic regression analysis. School Psychology International Journal, 2, 1-16. doi:10.1177/0020715211430373 
DiPerna, J. C., \& Elliott, S. N. (1999). The development and validation of the Academic Competence Evaluation Scale. Journal of Psychoeducational Assessment, 17, 207-225. doi:10.1177/073428299901700302

Elliott, S. N., \& Gresham, F. M. (2008). Social skills intervention guide. Bloomington, MN: Pearson Assessments.

Feitosa, F. B., Del Prette, Z. A. P., \& Del Prette, A. (2012). Social skills and academic achievement: The mediating function of cognitive competence. Temas em Psicologia, 20(1), 61-70.

Figueiredo, V. L. M. (2002). WISC-III: Escala de Inteligência Wechsler para Crianças - Adaptação brasileira da $3^{a}$ edição. São Paulo, SP: Casa do Psicólogo.

Floyd, R. G., Evans, J. J., \& McGrew, K. S. (2003). Relations between measures of Cattell-Horn-Carroll (CHC) cognitive abilities and mathematics achievement across the shool-age years. Psychology in the Schools, 40(2), 155-171. doi:10.1002/ pits. 10083

Freitas, L. C., \& Del Prette, Z. A. P. (2010a). Validade de critério do Sistema de Avaliação de Habilidades Sociais (SSRS-BR). Psicologia: Reflexão e Crítica, 23(3), 430-439. doi:10.1590/ S0102-79722010000300003

Freitas, L. C., \& Del Prette, Z. A. P. (2010b). Validade de construto do Sistema de Avaliação de Habilidades Sociais para crianças brasileiras com deficiência intelectual. Interamerican Journal of Psychology, 44(2), 312-320.

Freitas, L. C., \& Del Prette, Z. A. P. (2013). Habilidades sociais de crianças com diferentes necessidades educacionais especiais: Avaliação e implicações para intervenção. Avances en Psicología Latinoamericana, 31(2), 344-362.

Freitas, M. G. (2005). Desenvolvimento e avaliação de um programa de habilidades sociais com mães de crianças deficientes visuais (Tese de doutorado, Programa de Pós-Graduação em Educação Especial, Universidade Federal de São Carlos, SP, Brasil).

Freitas, M. L. P. F. (2012). Habilidades sociais e bem-estar subjetivo de crianças dotadas e talentosas (Tese de doutorado, Programa de Pós-Graduação em Psicologia, Universidade Federal de São Carlos, SP, Brasil).

Fumo, V. M. S. (2009). Habilidades sociais acadêmicas de crianças com alto e baixo desempenho acadêmico na interação com o professor (Dissertação de mestrado, Programa de Pós-Graduação em Educação Especial, Universidade Federal de São Carlos, SP, Brasil).

Gresham, F. M. (2009). Análise do comportamento aplicada às habilidades sociais. In Z. A. P. Del Prette \& A. Del Prette (Eds.), Psicologia das habilidades sociais: Diversidade teórica e suas implicações (pp. 17-66). Petrópolis, RJ: Vozes.

Gresham, F. M., \& Elliott, S. N. (1987). The relationship between adaptive behavior and social skills: Issues in definition and assessment. The Journal of Special Education, 21, 167-181. doi:10.1177/002246698702100115

Gresham, F. M., \& Elliott, S. N. (1990). Social skills rating system: Manual. Circle Pines, MN: American Guidance Service.

Gresham, F. M., \& Elliott, S. N. (2008). Social skills improvement system: Rating scales. Bloomington, MN: Pearson Assessments.

Gresham, F. M., Lane, K. L., MacMillan, D. L., \& Bocian, K. M. (1999). Social and academic profiles of externalizing and internalizing groups: Risk factors for emotional and behavioral disorders. Behavioral Disorders, 24(3), 231-245.

Guenther, Z. C. (2006). Dotação e talento: Reconhecimento e identificação. Revista Educação Especial (Santa Maria), 28(2), 195-208.
Hanley, G. P., Iwata, B. A., \& McCord, B. E. (2003). Functional analysis of problem behavior: A review. Journal of Applied Behavior Analysis, 36(2), 147-185. doi:10.1901/ jaba.2003.36-147

Hay, D. F., Hudson, K., \& Liang, W. (2010). Links between preschool children's prosocial skills and aggressive conduct problems: The contribution of ADHD symptoms. Early Childhood Research Quarterly, 25(4), 493-501. doi:10.1016/j. ecresq.2010.01.003

Hoerger, M. L., \& Mace, F. C. (2006). A computerized test of self-control predicts classroom behavior. Journal of Applied Behavior Analysis, 39(2), 147-159. doi:10.1901/ jaba.2006.171-04

Hosmer, D. W., \& Lemeshow, S. (2000). Applied logistic regression. New York: Wiley.

Hymel, S., Rubin, K. H., Rowden, R., \& LeMare, L. (1990). Children's peer relationships: Longitudinal prediction of internalizing and externalizing problems from middle to late childhood. Child Development, 61, 2004-2021. doi:10.1111/j.1467-8624.1990.tb03582.x

Individuals With Disabilities Education Act, 20 U.S.C. $§ 1400$ (2004). Retrieved September 21, 2009, from http://idea.ed.gov

Lehman, E. B., \& Erdwins, C. J. (2004). The social and emotional adjustment of young intellectually gifted children. In S. Moon (Ed.), Social/emotional issues, underachievement and counseling of gifted and talented students (pp. 1-8). Thousand Oaks, CA: Corwin.

Macintosh, K., \& Dissanayake, C. (2006). Social skills and problem behaviours in school aged children with Highfunctioning autism and Asperger's disorder. Journal of Autism and Developmental Disorders, 36, 1065-1076. doi:10.1007/ s10803-006-0139-5

Marchesi, A. (2004). Da linguagem da deficiência às escolas inclusivas. In C. Coll, A. Marchesi, \& J. Palácios (Eds.), Desenvolvimento psicológico e educação: Transtornos de desenvolvimento e necessidades educativas especiais (pp. 15-30). Porto Alegre, RS: Artmed.

Marton, I., Wiener, J., Rogers, M., Moore, C., \& Tannock, R. (2009). Empathy and social perspective taking in children with attention-deficit/hyperactivity disorder. Journal of Abnormal Child Psychology, 37, 107-118. doi:10.1007/ s10802-008-9262-4

Ministério da Educação. (2007). Política nacional de educação especial na perspectiva da educação inclusiva. Brasília, DF: Secretaria de Educação Especial.

Molina, R. C. M., \& Del Prette, A. (2007). Mudança no status sociométrico de alunos com dificuldades de aprendizagem. Psicologia Escolar e Educacional, 11(2), 299-310. doi:10.1590/ S1413-85572007000200009

Mrug, S., Hoza, B., Gerdes, A. C., Hinshaw, S., Arnold, L. E., Hechtman, L., \& Pelham, W. E. (2009). Discriminating between children with ADHD and classmates using peer variables. Journal of Attention Disorders, 12(4), 372-380. doi: $10.1177 / 1087054708314602$

Neef, N. A., Marckel, J., Ferreri, S. J., Bicard, D. F., Endo, S., Aman, M. G., ...Armstrong, N. (2005). Behavioral assessment of impulsivity: A comparison of children with and without attention deficit hyperactivity disorder. Journal of Applied Behavior Analysis, 38(1), 23-37. doi:10.1901/ jaba.2005.146-02

Organização Mundial da Saúde. (1997). Classificação de transtornos mentais e de comportamento da CID-10: Referência rápida. Porto Alegre, RS: Artes Médicas. 
Freitas, L. C. \& Del Prette, Z. A. P. (2014). Categorias de Necessidades Educacionais Especiais Enquanto Preditoras de Déficits em Habilidades Sociais na Infância.

Price, J. M., \& Zwolinski, J. (2010). The nature of child and adolescent vulnerability: History and definitions. In R. E. Ingram \& J. M. Price II (Eds.), Vulnerability to psychopathology: Risk across the lifespan (pp. 18-38). New York: Guildford Press.

Rocha, M. M. (2009). Programa de habilidades sociais educativas com pais: Efeitos sobre o desempenho social e academico de filhos com TDAH (Tese de doutorado, Programa de Pós-Graduação em Educação Especial, Universidade Federal de São Carlos, SP, Brasil).

Rubin, K. H., Coplan, R. J., \& Bowker, J. C. (2009). Social withdrawal in childhood. Annual Review of Psychology, 60 , 141-171. doi:10.1146/annurev.psych.60.110707.163642

Schalock, R. L., Borthwick-Duffy, S. A., Bradley, V. J., Buntinx, W. H. E., Coulter, D. L., Craig, E. M., ...Yeager, M. H. (2010). Intellectual disability: Definition, classification, and systems of supports. Washington, DC: American Association on Intellectual and Developmental Disabilities.

Schopler, E., Reichler, J. R., \& Renner, C. (1988). CARS: The Childhood Autism Rating Scale. Los Angeles, CA: Westerm Psychological Services.

Sorlie, M. A., Hagen, K. A., \& Ogden, T. (2008). Social competence and antisocial behavior: Continuity and distinctiveness across early adolescence. Journal of Research on Adolescence, 18(1), 121-144. doi:10.1111/j.1532-7795.2008.00553.x

Souza, T. M. P. (2008). Inclusão de crianças com deficiência auditiva na escola regular: Uma análise sob a perspectiva das habilidades sociais (Dissertação de mestrado, Programa de Pós-Graduação em Educação Especial, Universidade Federal de São Carlos, SP, Brasil).

Stein, L. M. (1994). Teste de desempenho escolar: Manual para aplicação e interpretação. São Paulo, SP: Casa do Psicólogo.

Tabachnick, B. G., \& Fidell, L. S. (2007). Using multivariate statistics $\left(5^{\text {th }}\right.$ ed.). New York: Allyn and Bacon.

Thomas, L. B., Shapiro, E. S., DuPaul, G. J., Lutz, J. G., \& Kern, L. (2011). Predictors of social skills for preschool children at risk for ADHD: The relationship between direct and indirect measurements. Journal of Psychoeducational Assessment, 29(2), 114-124. doi:10.1177/0734282910378478

Versteynen, L. (2001). Issues in the social and emotional adjustment of gifted children: What does the literature say? The New Zealand Journal of Gifted Education, 13(1), 1-8.

Wauters, L. N., \& Knoors, H. (2008). Social integration of deaf children in inclusive settings. Journal of Deaf Studies and Deaf Education, 13(1), 21-36. doi:10.1093/deafed/enm028 